\title{
ERRATUM
}

\section{Integrated Approaches to Address the Social Determinants of Health for Reducing Health Inequity}

Franৎoise Barten, Diana Mitlin, Catherine Mulholland, Ana Hardoy, and Ruth Stern

"Develppment" should be "Development" in affiliation (2) in the xml for this article.

Barten is with the Radboud University Nijmegen, UMCN, Institute for International Health, Department of Public Health, PO Box 9101-117, 6500 HB, Nijmegen, The Netherlands; Mitlin is with the University of Manchester and the International Institute for Environment and Development, London, UK; Mulholland is with the World Health Organization, Geneva, Switzerland; Hardoy is with the International Institute for Environment and Development, Buenos Aires, Argentina; Stern is with the School of Public Health, University of Western Cape, Bellville, South Africa; Mulholland is with the II, Route de Provins, 74940, Annecy le Vieux, France.

Correspondence: Fraņoise Barten, Radboud University Nijmegen, UMCN, Institute for International Health, Department of Public Health, PO Box 9101-117, 6500 HB, Nijmegen, The Netherlands. (E-mail: f.barten@aig.umcn.nl)

The online version of this article can be found at http://dx.doi.org/10.1007/s11524-007-9173-7. 